\title{
Transcriptional regulation of 1-cys peroxiredoxin by the proto-oncogene protein DEK
}

\author{
DONG-WOOK KIM ${ }^{2}$, JI-YOUNG KIM ${ }^{1}$, SULJI CHOI ${ }^{1}$, SANGMYUNG RHEE ${ }^{1}$, \\ YOONSOO HAHN $^{1}$ and SANG-BEOM SEO ${ }^{1}$ \\ ${ }^{1}$ Research Center for Biomolecules and Biosystems, Department of Life Science, College of Natural Sciences, \\ Chung-Ang University, Seoul 156-756; ${ }^{2}$ Department of Biomedical Science, College of Life Science, \\ CHA University, Seongnam 463-836, Korea
}

Received April 22, 2010; Accepted July 26, 2010

DOI: $10.3892 / \mathrm{mmr} .2010 .346$

\begin{abstract}
The nuclear phosphoprotein DEK is abundantly present in cells and is implicated in diseases including leukemia and autoimmune disorders. DEK has three highly acidic amino acid domains and inhibits histone acetyltransferase activity by binding directly to histones. In a previous study, differentially regulated proteins under DEK knockdown conditions, including the up-regulated protein 1-cys peroxiredoxin (1-cys Prx), were identified by proteome analysis. Here, an in vivo reporter assay with short hairpin RNA-mediated DEK knockdown revealed that DEK negatively regulated 1-cys Prx transcription, and that the NF- $\mathrm{BB}$ subunit $\mathrm{p} 65$ had a synergistic effect on this DEK-mediated repression. Both proteins are recruited to the 1-cys Prx promoter region and regulate its transcription. Our study demonstrates that DEK modulates the transcriptional regulation of the target gene through protein interaction with other regulatory proteins.
\end{abstract}

\section{Introduction}

The DEK protein was first identified in a specific chromosomal translocation $\mathrm{t}(6 ; 9)(\mathrm{p} 23 ; \mathrm{q} 34)$ in an acute myelogenous leukemia, which results in the formation of a fusion gene with the CAN nucleoporin protein NUP214 (1). This translocated fusion gene makes a fusion protein, in which part of the N-terminal (1-349 amino acids) of DEK is fused with part of the C-terminal (813-2,090 amino acids) of CAN. The DEK protein, as a phosphoprotein, is an abundantly and ubiquitously expressed nuclear protein with two functional domains (SAP and DNA binding/multimerization), several phosphorylation sites and three highly acidic domains $(2,3)$. DEK binds DNA and induces DEK-DEK multimerization in a phosphorylation-dependent manner (4). A recent report

Correspondence to: Professor Sang-Beom Seo, Research Center for Biomolecules and Biosystems, Department of Life Science, College of Natural Sciences, Chung-Ang University, Seoul 156-756, Korea E-mail: sangbs@cau.ac.kr

Key words: DEK, 1-cys peroxiredoxin, p65, transcription showed that a highly acidic domain in DEK inhibits histone acetyltransferase (HAT) activity through histone binding (2). It has been revealed that DEK influences chromatin remodeling through the alteration of chromatin topology $(5,6)$. DEK also regulates transcriptional activity by recruiting transcriptional co-repressors, such as hDaxx and histone deacetylase-2, to chromatin (7).

DEK is also involved in carcinogenesis and autoimmune diseases, such as systemic lupus erythematosus, juvenile rheumatoid arthritis and ataxia telangiectasia (8). A recent proteomic analysis revealed that DEK knockdown up-regulated 1-cys peroxiredoxin (1-cys Prx) (Prx 6) and caused the hyperacetylation of histones around the 1-cys Prx promoter (9).

1-cys Prx is one of six Prx isoforms, and is expressed in various tissues - in particular liver tisses - related to the protection of cellular oxidative stresses (10).

$\mathrm{NF}-\kappa \mathrm{B}$ is a transcription factor with multiple biological functions, including immune and inflammatory response, cell growth and differentiation, and the suppression of apoptosis (11). The NF- $\kappa \mathrm{B}$ family consists of five members, p65/RelA, p50 (p105), p52 (p100), RelB and c-Rel (12). A previous study showed that the p65 subunit of NF-кB interacts with DEK (13).

In this study, we identified the negative regulatory role of DEK in 1-cys Prx transcription: its knockdown rescues the transcriptional activation of 1-cys Prx through the p65 subunit of NF- $\mathrm{B}$. Additionally, we showed that the p65 subunit of $\mathrm{NF}-\kappa \mathrm{B}$ synergistically represses 1 -cys Prx by recruitment to the promoter.

\section{Materials and methods}

Plasmid constructs. For the reporter assay, the following pCMX plasmid constructs were used: CMX-DEK (wild-type), CMX-DEK $\Delta 290$ and CMX-DEK $\Delta 200$ (2). The p65 subunit coding region was obtained by PCR amplification using the following specific primers: BamHI site-linked 5'-CGCGGA TCCATGGACGAACTGTTCCCCCTC-3' (forward), and XhoI site-linked 5'-CGCCTCGAGGGAGCTGATCTGACTCAG CAG-3' (reverse). Gel-purified PCR product was cloned into the modified pcDNA6-myc vector by BamHI-XhoI enzyme digestion, which has a triple-tag sequence (myc, his and HA). 
Knockdown of endogenous DEK. To suppress DEK expression, a vector-based RNAi method was used. pSM2c-DEK expressing DEK specific short hairpin RNAs (nucleotides 654-674 from NM_003472), referred to as shRNA, was purchased from Open Biosystems (Hunsville, AL, USA).

Cell culture and luciferase reporter assay. HeLa cells were grown in Dulbecco's modified Eagle's medium (Invitrogen, Carlsbad, CA, USA) containing $10 \%$ heat-inactivated fetal bovine serum (Invitrogen) and $0.05 \%$ penicillin-streptomycin (Invitrogen) at $37^{\circ} \mathrm{C}$ in a $5 \% \mathrm{CO}_{2}$ humidified atmosphere. HeLa cells were seeded in 48-well dishes and transfected by lipofectamine 2000 (Invitrogen) with pGL3-1-cys Prx $(100 \mathrm{ng})$ in the absence or presence of pcDNA6-p65-myc $(50,100,150$ or $200 \mathrm{ng})$ or $\operatorname{shDEK}(50,100,150$ or $200 \mathrm{ng})$, and each of the deletion mutants (wild-type, $\Delta 290$ and $\Delta 200$; $50,100,200$ or $300 \mathrm{ng}$ ). To demonstrate the role of DEK in p65-mediated transcriptional regulation, pGL3-1-cys Prx (100 ng) was transfected with pcDNA6-p65-myc and further transfected with increasing amounts (50 and $100 \mathrm{ng}$ ) of shDEK plasmid. Luciferase activity was measured by the addition of $20 \mu \mathrm{l}$ luciferin as a substrate into $80 \mu \mathrm{l}$ of cell lysates using the Glomax luminometer (Promega, Pittsburg, PA, USA). Each value is the mean of four replicates from a single assay. The results are representative of at least three independent experiments.

Chromatin immunoprecipitation analysis. HeLa cells were transfected with CMX-DEK $(4.8 \mu \mathrm{g})$ or shDEK $(3 \mu \mathrm{g})$ plasmids and harvested at 48 or $72 \mathrm{~h}$ post-transfection. The cells were crosslinked with $1 \%$ formaldehyde in medium for $10 \mathrm{~min}$ at $37^{\circ} \mathrm{C}$ followed by the addition of $125 \mathrm{mM}$ glycine for $5 \mathrm{~min}$ at room temperature, and were then scraped into SDS lysis buffer. Cell lysates were sonicated and diluted for Chromatin immunoprecipitation (ChIP) with the antibodies: anti-p65 $(5 \mu \mathrm{g})$, anti-myc $(5 \mu \mathrm{g})$ and anti-DEK $(5 \mu \mathrm{g})$ (Santa Cruz Biotechnology,Paso Robles,CA,USA).Theimmunoprecipitates were eluted and reverse crosslinked. DNA fragments were purified and quantitated using PCR amplification. For the analysis of the 1-cys Prx promoter, intron and exon regions, the following PCR primers were designed: promoter region (position -911 to -672 from translation start site), 5'-GTT GACCTGCACACAGTAGGTCTC-3' (forward) and 5'-CCT ACAGTGGAGTGGAGTGACTGCT-3' (reverse); final intron region (position 9,353 to 9,708), 5'-GTCATGGCTGTAAAA GTACTGGTG-3' (forward) and 5'-CACTGGAATGGAAGT TCTATGAGGG-3' (reverse); final exon (position 9,989 to 10,347), 5'-GCTTGGAGAAGAAGCTGCAGAA-3' (forward) and 5'-CTATCCCCATCCTATTGAAAGAC-3' (reverse).

\section{Results and Discussion}

Transcriptional regulation of 1-cys Prx by DEK. Previously, we identified differential proteomic profiles in DEK knockdown HeLa cells (9), among them the regulated protein 1-cys Prx, which was up-regulated. The regulatory mechanism of 1-cys Prx was further investigated in this study. To investigate whether DEK regulates the transcription of 1-cys Prx, we performed a luciferase reporter assay, in which the human 1-cys Prx promoter region (positions -51 to $-1,501$ ) was cloned

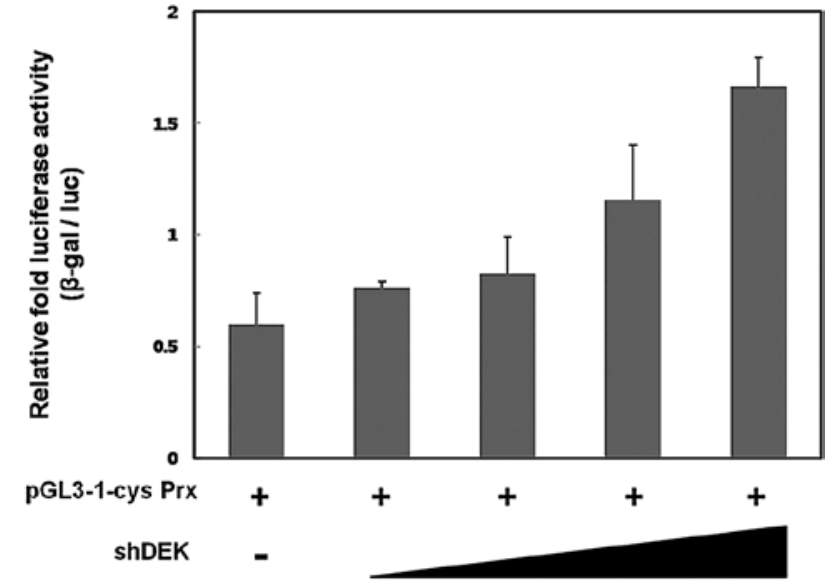

Figure 1. Knockdown of endogenous DEK enhances 1-cys Prx transcriptional activity. HeLa cells were transfected with 1-cys Prx reporter (100 ng) and $\operatorname{shDEK}(0,50,100,150$ or $200 \mathrm{ng})$. Relative luciferase activity was determined at $48 \mathrm{~h}$ post-transfection. Bars represent the average of three independent assays and error bars represent the \pm SD.

into pGL3-basic vector. The transcriptional activity of 1-cys Prx was initially measured, and an increasing amount of shDEK was co-transfected. As expected, the knockdown of DEK enhanced 1-cys Prx transactivation in a dose-dependent manner (Fig. 1). This reporter assay indicates that the transcription of 1-cys Prx is regulated by DEK.

C-terminus HAT inhibitory domains are responsible for DEK-mediated transcriptional repression of 1-cys Prx. We previously demonstrated that DEK inhibits histone acetylation by p300/CBP or PCAF and directly binds to histones (2). Moreover, the acidic domains of DEK are critical for HAT inhibitory activity and histone binding affinity. To further investigate the functional role of acidic domains in the transcriptional regulation of 1-cys Prx, reporter assays were performed using DEK deletion mutants. A series of C-terminal acidic domain deletion mutants, DEK- $\Delta$ C290 and DEK- $\Delta$ C200, were used in the reporter assay (Fig. 2A). Recently, we showed that the deletion mutant DEK- $\Delta$ C 200 , which has lost the $\mathrm{C}$-terminus acidic domains, showed very weak HAT inhibitory activity (Fig. 2A) (2). Consistent with the finding that shDEK mediated transactivation, the overexpression of wild-type DEK significantly repressed 1-cys Prx transcriptional activity (Fig. 2B). Transient transfection of DEK- $\Delta$ C290 reversed DEK-mediated transcriptional repression, and DEK- $\Delta \mathrm{C} 200$, which lost both C-terminal acidic domains, further rescued the repression of 1-cys Prx (Fig. 2B). These results are consistent with the acidic domains-mediated HAT inhibitory and transcriptional repression activities of DEK, and indicate the importance of acidic domains in the C-terminus.

Transcriptional repression of 1-cys Prx by the p65 subunit of $N F-\kappa B$. Bioinformatical analysis was performed with the 1-cys Prx promoter region using TESS (http://www.cbil. upenn.edu/cgi-bin/tess/tess) to identify the transcription factors that affect 1-cys Prx gene expression. By a computational search, the predicted NF- $\mathrm{KB}$ binding site in the 
A

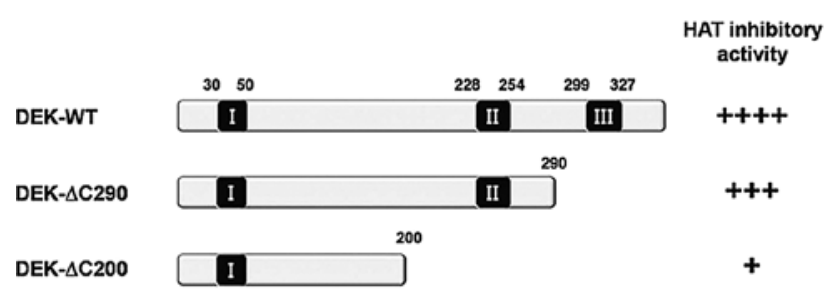

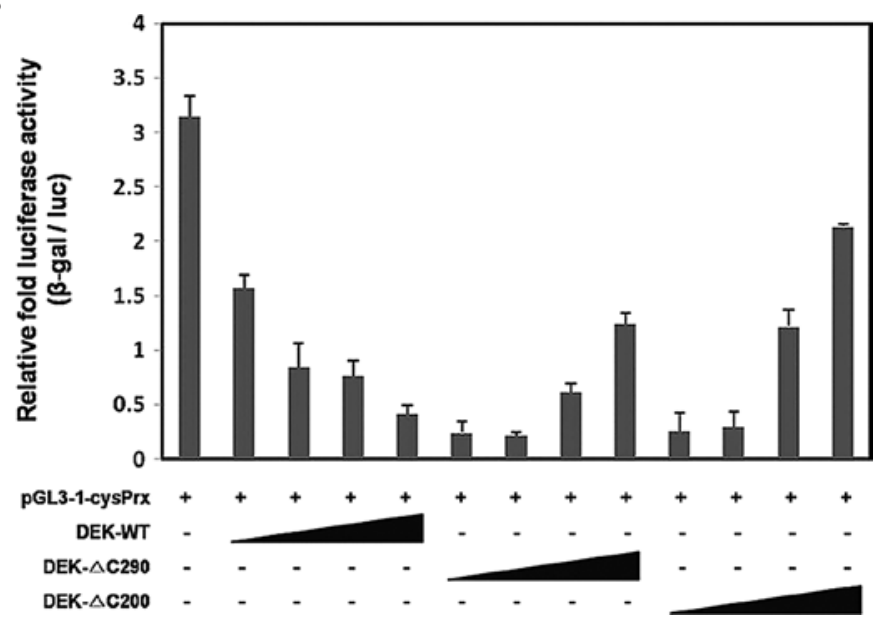

Figure 2. The C-terminus acidic domain of DEK is key in the transcriptional repression of 1-cys Prx. (A) Schematic diagram of DEK deletion mutants. Black boxes indicate acidic domains in the DEK protein. The HAT inhibitory activity of each mutant is indicated. Briefly, HAT inhibitory activity is also classified on a scale from ++++ (high activity) to + (little activity, $20 \%$ of wild-type). (B) HeLa cells were transfected with DEK wild-type (DEK-WT) and the deletion constructs DEK- $\Delta C 290$ and $-\Delta C 200(50,100,200$ and $300 \mathrm{ng}$ each) together with 1-cys Prx reporter (100 ng). Relative luciferase activity was determined at $48 \mathrm{~h}$ post-transfection. Bars represent the average of three independent assays and error bars represent the $\pm \mathrm{SD}$.

A

1 ATGAaAaCOC AaATCACGGT ATGTGAGTCA CTACTCTCCA CATTOCCATO CCCCTOCCTT AAACCTOCCA GCCCCATCCA

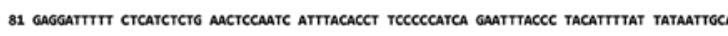
161 tataAataTG CCTTCCCTG AaCAGCAGGG ACTGTGTTTC TCCACTGTAT GGCCTGGCAC AGTGTCCAGC ACACAGCAGG 241 CAAAGCAaGA GTTACTGCAT GAGCCAGTG CATCATCCAA ACGATAGCTA CCATTTCCAG AGCACCTACC GTGGCTGAC 321 TAGTTTACCT ACATTATCCT GGTGGCTTTC CCAGACCAGA CCACCCAAAC CAAGGCAGCC CCCCAGTCAC CCTCTAGGCA 401 CATAACCCTA TTTTAGTTCT CTGAATACGA CTGATTGCTO TCTAACATAT TTTGGTTTGT GTGCTTATTG TCTGTCACCO

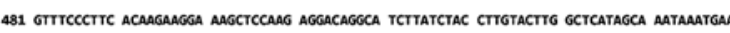
561 TCTAAGCCGC CCACCTCCCT GGGTGACAGA GTTGACCTGC ACACAGTAGG TCTCAaAGaG TATGTTTTAA ATTTTATATT

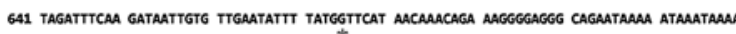

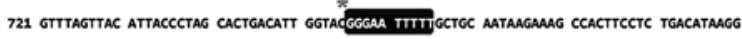

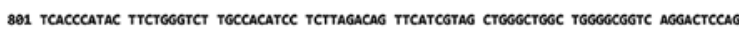
881 ACGCTCACAC TARAAGAGGG GTGGTAGTCG AGCAGTCACT CCACTCCTCT GTAGGGAGCT GGCCGCGAGT GGGTGCGCAG 961 CAGGGTGCGC GCGGACCCGT GCAGCGCCCA GGGCGGCTGT GCGAAGCCGC CGCAGCCTGC GCGCCCAGGC GATTCTCCAC 1041 CTTGGGSCTC GCCTTCCTOC CGCGTTCCTC TTCCTTCCAT CCCTAAAGCG CGTACGCCCT GCAGAGTCAA ACCTGGCECA 1121 TCCTCAAGCT TCCAGGGGGC AaCGTGACCG AGCCCCGCAT CACGTGTGCA GAGACGGCCT ATAGTGTCTG AAGGATGCCA

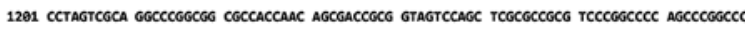

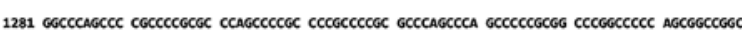

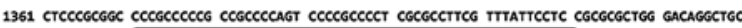

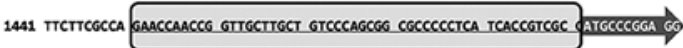

Putative NF-KB binding site $3^{*}$-UTR
B

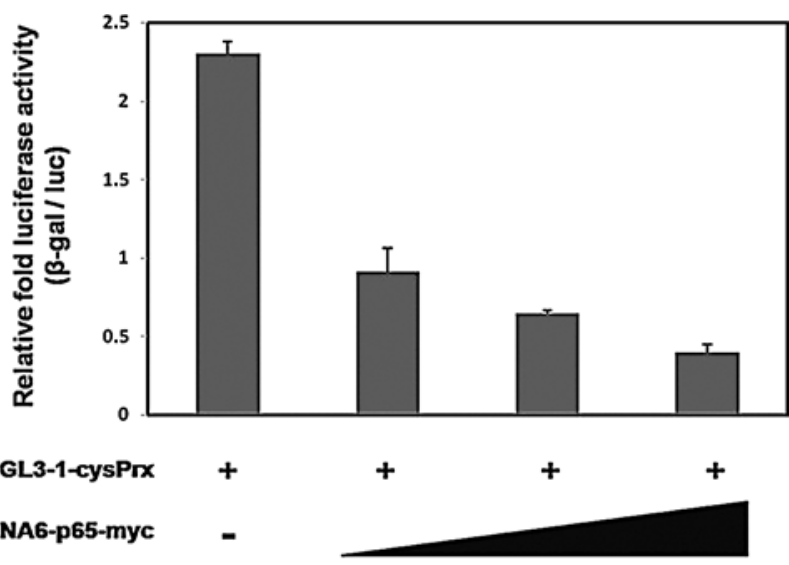

C

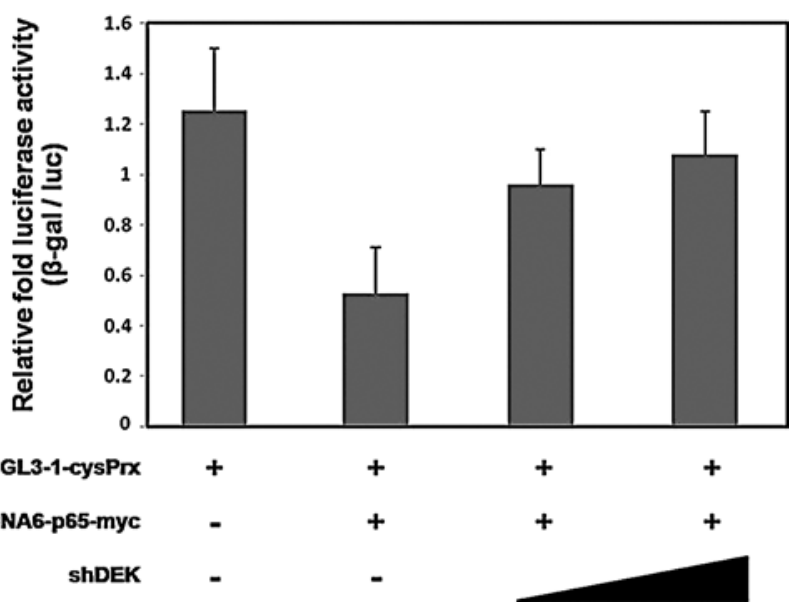

Figure 3. Transcriptional repression of 1-cys Prx by p65 and DEK. (A) The asterisks indicate the putative binding sites, and the arrow box designates the coding region of 1-cys Prx as determined by the TESS program. The underlined sequence in the box is the 3' untranslated region. (B) HeLa cells were transfected with 1-cys Prx reporter (100 ng) and pcDNA6-p65-myc (0, 50, 100 and $150 \mathrm{ng}$ ). Relative luciferase activity was determined at $48 \mathrm{~h}$ post-transfection Bars represent the average of three independent assays and error bars represent the \pm SD. (C) HeLa cells were transfected with pcDNA6-p65-myc (100 ng) and shDEK (50 and $100 \mathrm{ng}$ ) together with 1-cys Prx reporter (100 ng). Relative luciferase activity was determined at $48 \mathrm{~h}$ post-transfection. Bars represent the average of three independent assays and error bars represent the \pm SD. 
A

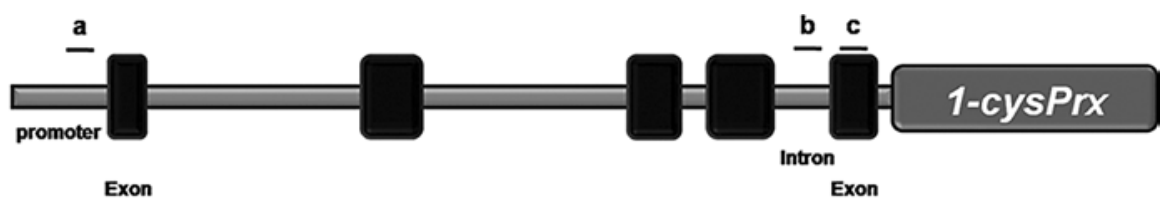

B

i) promoter region:

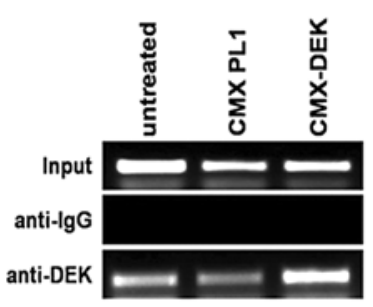

ii) Final intron:

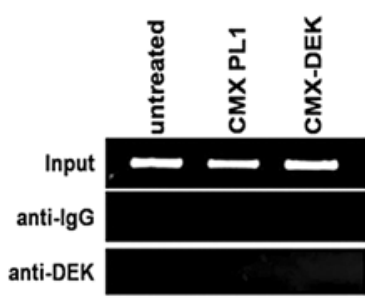

iii) Final exon:

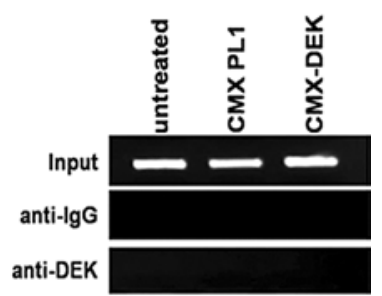

C

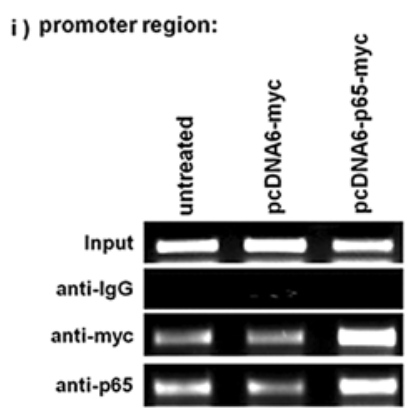

ii) Final intron:

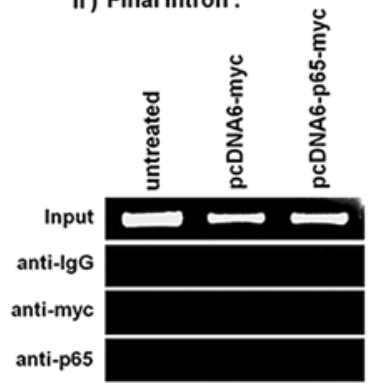

iii) Final exon :

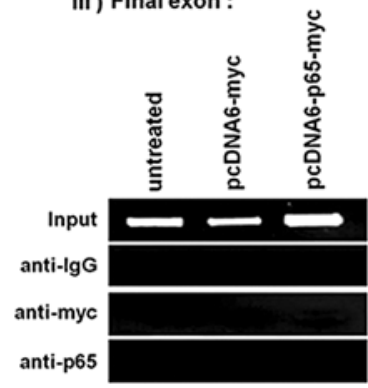

Figure 4. Occupancy of DEK and the p65 subunit in the 1-cys Prx promoter region. (A) Schematic diagram of the primer pairs used for ChIP analysis. Black boxes represent the 1-cys Prx exons and the gray bar indicates the intron. (B and C) HeLa cell lysates were transfected with CMX-PL1, CMX-DEK, pcDNA6-myc or pcDNA6-p65-myc and analyzed by ChIP using mouse IgG, anti-DEK antibodies (B) or mouse IgG, anti-p65 and anti-myc antibodies (C). The immunoprecipitated DNA was amplified by PCR using promoter (i), final intron (ii) and final exon (iii) specific primer pairs.

1-cys Prx promoter region was positioned at -737 to -746 from the transcriptional start site (Fig. 3A). This putative NF- $\mathrm{KB}$ binding site is also present in the pGL3-1-cys Prx reporter vector. A previous report demonstrated that HDACs modulate the transcriptional activity of NF- $\mathrm{kB}$ through protein-protein interaction (14). DEK was characterized as a p65 interacting protein by a yeast-two hybrid experiment, and shown to result in the inhibition of p65-mediated transcriptional activity (13). The fact that DEK interacts with p65 and that both proteins are involved in transcriptional repression prompted us to investigate whether p65 protein has a role in DEK-mediated 1-cys Prx transcriptional repression. The reporter assay was first carried out with 1-cys Prx promoter and pcDNA6-p65-myc. Increasing concentrations of p65 caused the transcriptional activity of 1-cys Prx to be down-regulated (Fig. 3B).

NF- $\mathrm{KB}$ regulates many cellular processes through the transcriptional activation of target genes. However, Shaw et al suggested that NF- $\mathrm{KB}$ may also act as a transcriptional repressor via interaction with HDACs (15). Transcriptional repression of 1-cys Prx by p65 was reversed by co-transfection with shDEK (Fig. 3C). Our results indicate that p65 and DEK have a synergistic effect on the transcriptional repression of 1-cys Prx.
Recruiting of DEK and p65 in the 1-cys Prx promoter. To further demonstrate that both DEK and p65 are recruited to the 1-cys Prx promoter region, a ChIP assay was performed. As expected, when DEK and p65 were overexpressed, the 1-cys Prx promoter region was occupied by both DEK and p65 as compared to untreated and empty vector transfected cells (Fig. 4B-i and $\mathrm{C}-\mathrm{i})$. By contrast, the 1-cys Prx final intron and final exon were not occupied by either protein (Fig. 4B-ii and iii and C-ii and iii)]. These ChIP assay results indicate that both the DEK and p65 proteins are recruited to the 1-cys Prx promoter region and negatively regulate its transcription. It is possible that p65 binds directly to DNA and recruits DEK protein as a transcriptional repressor through protein-protein interaction.

In this study, we identified the functional role of DEK in 1-cys Prx transcription. DEK functions as a transcriptional repressor of 1-cys Prx by cooperating with the p65 subunit of NF- $\kappa B$. Further studies are required to elucidate the detailed mechanisms of DEK and p65-mediated transcriptional regulation of 1-cys Prx, and its physiological effects.

\section{Acknowledgements}

This study was supported by the Chung-Ang University Research Scholarship Grant (2009). 


\section{References}

1. Von Lindern M, Fornerod M, van Baal S, et al: The translocation $(6 ; 9)$, associated with a specific subtype of acute myeloid leukemia, results in the fusion of two genes, dek and can, and the expression of a chimeric, leukemia-specific dek-can mRNA. Mol Cell Biol 12: 1687-1697, 1992.

2. Ko SI, Lee IS, Kim JY, Kim SM, Kim DW, Lee KS, Woo KM, Baek JH, Choo JK and Seo SB: Regulation of histone acetyltransferase activity of p300 and PCAF by proto-oncogene protein DEK. FEBS Lett 580: 3217-3222, 2006.

3. Waldmann T, Scholten I, Kappes F, Hu HG and Knippers R: The DEK protein - an abundant and ubiquitous constituent of mammalian chromatin. Gene 343: 1-9, 2004.

4. Kappes F, Scholten I, Richter N, Gruss C and Waldmann T: Functional domains of the ubiquitous chromatin protein DEK. Mol Cell Biol 24: 6000-6010, 2004.

5. Alexiadis V, Waldmann T, Andersen J, Mann M, Knippers R and Gruss C: The protein encoded by the proto-oncogene DEK changes the topology of chromatin and reduces the efficiency of DNA replication in a chromatin-specific manner. Genes Dev 14 1308-1312, 2000.

6. Waldmann T, Eckerich C, Baack M and Gruss C: The ubiquitous chromatin protein DEK alters the structure of DNA by introducing positive supercoils. J Biol Chem 277: 24988-24994, 2002.

7. Hollenbach AD, McPherson CJ, Mientjes EJ, Iyengar R and Grosveld G: Daxx and histone deacetylase II associate with chromatin through an interaction with core histones and the chromatin-associated protein DEK. J Cell Sci 115: 3319-3300, 2002.
8. Carro MS, Spiga FM, Quarto M, Di Ninni V, Volorio S, Alcalay $\mathrm{M}$ and Muller H: DEK expression is controlled by E2F and deregulated in diverse tumor types. Cell Cycle 5: 1202-1207, 2006.

9. Kim DW, Chae JI, Kim JY, Pak JH, Koo DB, Bahk YY and Seo SB: Proteomic analysis of apoptosis related proteins regulated by proto-oncogene protein DEK. J Cell Biochem 106: 1048-1059, 2009.

10. Manevich Y, Sweitzer T, Pak JH, Feinstein SI, Muzykantov V and Fisher AB: 1-Cys peroxiredoxin overexpression protects cells against phospholipid peroxidation-mediated membrane damage. Proc Natl Acad Sci 99: 11599-11604, 2002.

11. Hatada EN, Krappmann D and Scheidereit C: NF-kappaB and the innate immune response. Curr Opin Immunol 12: 52-58, 2000.

12. Karin M and Ben-Neriah Y: Phosphorylation meets ubiquitination: the control of NF-kappaB activity. Annu Rev Immunol 18 621-663, 2000.

13. Sammons M, Wan SS, Vogel NL, Mientjes EJ, Grosveld G and Ashburner BP: Negative regulation of the RelA/p65 transactivation function by the product of the DEK proto-oncogene. J Biol Chem 281: 26802-26812, 2006.

14. Yeung F, Hoberg JE, Ramsey CS, Keller MD, Jones DR, Frye RA and Mayo MW: Modulation of NF-kappaB-dependent transcription and cell survival by the SIRT1 deacetylase. Embo J 23: 2369-2380, 2004.

15. Shaw J, Zhang T, Rzeszutek M, Yurkova N, Baetz D, Davie JR and Kirshenbaum LA: Transcriptional silencing of the death gene BNIP3 by cooperative action of NF-kappaB and histone deacetylase 1 in ventricular myocytes. Circ Res 99: 1347-1354, 2006. 